\title{
A Simple Approach to Dynamic Optimisation of Flexible Optical Networks with Practical Application
}

\author{
Vic Grout \\ Applied Research in Computing Laboratory (ARCLab), Wrexham Glyndŵr University, Wrexham, \\ Wales, LL11 2AW, UK; v.grout@glyndwr.ac.uk; Tel.: +44-1978-293203 \\ Academic Editor: Dino Giuli \\ Received: 20 April 2017; Accepted: 17 May 2017; Published: 23 May 2017
}

\begin{abstract}
This paper provides an initial introduction to, and definition of, the 'Dynamically Powered Relays for a Flexible Optical Network' (DPR-FON) problem for opto-electro-optical (OEO) regenerators used in optical networks. In such networks, optical transmission parameters can be varied dynamically as traffic patterns change. This will provide different bandwidths, but also change the regeneration limits as a result. To support this flexibility, OEOs ('relays') may be switched on and off as required, thus saving power. DPR-FON is shown to be NP-complete; consequently, solving such a dynamic problem in real-time requires a fast heuristic capable of delivering an acceptable approximation to the optimal configuration with low complexity. In this paper, just such an algorithm is developed, implemented, and evaluated against more computationally-demanding alternatives for two known cases. A number of real-world extensions are considered as the paper develops, combining to produce the 'Generalised Dynamically Powered Relays for a Flexible Optical Network' (GDPR-FON) problem. This, too, is analysed and an associated fast heuristic proposed, along with an exploration of the further research that is required.
\end{abstract}

Keywords: flexible optical networks; FONs; opto-electro-optical regenerators; OEOs; optimisation problems; NP-complete; dynamic relay optimisation; DPR-FON; algorithmic complexity; heuristics; GDPR-FON

\section{Introduction and Background}

Optimising the location of opto-electro-optical (OEO) regenerators (hereafter referred to as relays) in optical networks (ONs) has, for much of the history of ONs, been considered a static problem [1]. Such devices have, until recently, been expensive to both install and operate. In consequence, optimisation of relay placement has been performed offline, with complex algorithms, leading to solutions to be implemented as part of a fixed, long-term network structure [2]. Recent years, however, have seen two significant developments in the field:

The introduction of flexible optical network (FON) technology has led to ONs having the ability to vary transmission parameters to support changing traffic requirements. By modulating across larger bandwidths, FONs can achieve higher bitrates at the expense of shorter transmission distances (whereas, conversely, greater transmission distances can be achieved for lower bitrates) [3].

The production and installation of OEOs (relays) has fallen sharply due to both improved manufacturing processes and refinements to carrier/switching technologies, although running costs, in terms of power consumption, are still high [4]. Consequently, installing relays in multiple locations but using (powering) them selectively (as, and when, required) may lead to greater efficiencies.

Describing the engineering principles behind these advances is beyond the scope of this work [5]. However, in essence, the new technology (1) makes the optimisation of relay management a dynamic problem [6] whilst the emphasis on running costs (2) redefines its objective [7]. As a result, optimising 'placement' of relays becomes an optimisation of relay 'use'. Higher traffic will mean larger bandwidths, 
shorter transmission limits and, thus, the need for more frequent regeneration and greater numbers of relays.

The next section considers comparable optimisation problems, which yield some insight into the task at hand. Section 3 gives the initial formulation and complexity discussion. Useful related approaches are considered in Section 4, and these lead to the proposed algorithm in Section 5, which also provides the proof of NP-completeness. Section 6 tests the algorithm with two known library networks. In Section 7, the problem, and its solution, are generalised and the complexity considered once more. Section 8 suggests future work for other researchers. The paper's overall structure, switching as it does between definition and solution, may appear odd, but this approach is considerably shorter than dealing with each thread independently in turn. For the same reason, the notation is also slightly informal in places.

\section{Related Problems}

Some aspects of the efficient management [8] and optimisation [9] of FONs have been recently documented as the technology and its benefits have become more widely known. However, until now, the approach has been to define a multi-objective problem, involving several aspects of traffic management, in turn requiring expensive optimisation [10]. In [3], for example, a complex off-line linear programming formulation is given, which requires considerable computing power to solve. We propose two significant variations to this general approach in this paper:

1. We do not consider complex traffic management, such as dimensioning [11], routing [12], or shaping [13], in our formulation, focusing solely on relay use.

2. Instead, we require a dynamic, computationally-inexpensive solution to the problem, which can be implemented in real-time in response to changing traffic patterns.

Although (1) simplifies the problem, (2) significantly increases the challenge.

For completeness, relevant optimisation problems and solutions from other fields (in networking and graph theory) include the Minimum Dominating Set (MDS) [14] and the Minimum Connected Dominating Set (MCDS) [15], Minimum Spanning Tree (MST) [16], Maximum Leaf Spanning Tree (MLSP) [17], and Shortest Path (SPP) [18] problems. We draw on components of all of these in this paper, described, where appropriate, in what follows.

\section{Formulation of the Problem}

In this paper, we use a simple model of the problem, with possible extensions considered in Section 7. Some of these extensions are, in fact, straightforward, but are omitted initially to minimise the requirements of the initial notation. Additionally, in what follows, we use a number of standard logical, set-theoretic, and optimisation-based concepts and abbreviations, namely: there are $n$ nodes in the $\mathrm{ON} / \mathrm{FON}$, numbered $1,2, \ldots, n-1, n$; the logical operators 'AND' and 'OR' are represented by ' $\wedge$ ' and ' $V$ ' respectively; 'Such that' is abbreviated to 's.t.' and 'TRUE' and 'FALSE' to ' $T$ ' and ' $F$ '; $|S|$ represents the size ('cardinality') of the set $S$; and, finally, the universal and existential operators ' $\forall$ ' and ' $\exists$ ' ('for all' and 'there exists') refer explicitly to the set of nodes $1,2, \ldots, n-1, n$ (hereafter omitted).

For a given node set and link configuration (Figure 1), define the relay vector, $\underline{r}$, as: $\underline{r}=\left(r_{k}\right)$ where $\forall k, r_{k}=T$ if the relay at node $k$ is switched on (powered); $r_{k}=F$ otherwise. $\underline{r}$ defines relay use for a given configuration. Initially, we suppose that relays are placed at all nodes but are only activated when required (see Ext1 in Section 7). Let $m$ be the maximum number of hops for which an optical signal can be transmitted without the need for regeneration by an OEO (relay). A hop is a single link between adjacent nodes (see Ext2 in Section 7). The critical feature of a FON is that changing transmission parameters, to dynamically increase or decrease bandwidth, will cause $m$ to vary ( $m$ will decrease with higher bitrates and increase with lower bitrates). Initially, we take $m$ to be a single (but not static) parameter across the FON (see Ext3 in Section 7). As $m$ varies, different requirements will be placed (dynamically) on relay placement, $\underline{r}$. In the FON fragment in Figure 2a, for example, values for $m$ of 1 , 
2 , and 3 cannot be sustained, whereas an $m$ value of 4 can. In Figure $2 \mathrm{~b}$, now with $r_{k}=T$, values of $m=$ $4, m=3$, and $m=2$ can all be sustained, and only $m=1$ cannot.

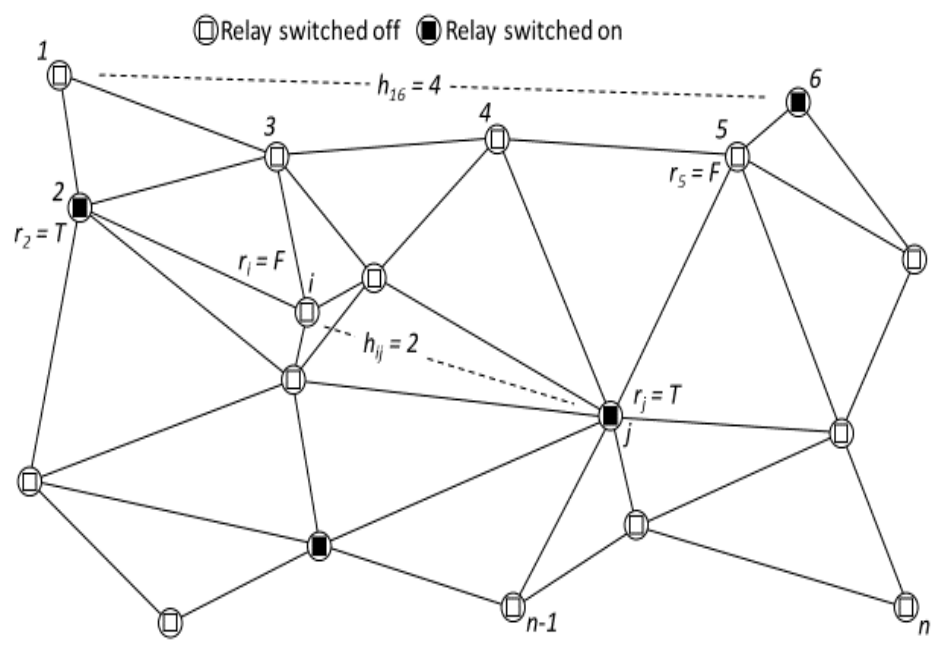

Figure 1. A flexible optical network (FON) with OEOs (relays) at all locations.

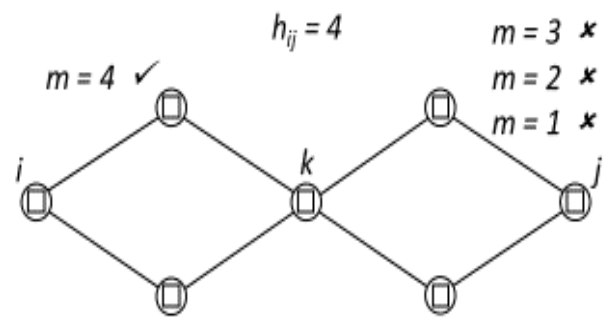

(a)

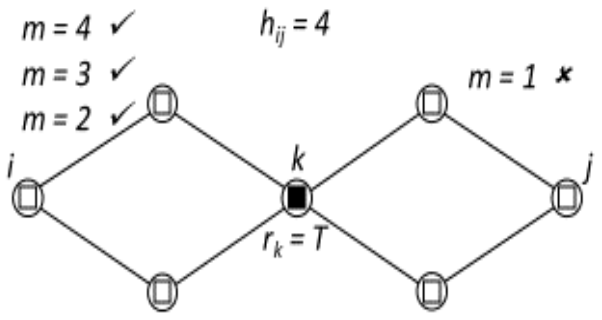

(b)

Figure 2. Hops and transmission limits.

The cost of a solution, $\underline{r}$, is then the number of relays switched on in any given configuration. $C(\underline{r})=\left|\left\{k: r_{k}=T\right\}\right|$ (the cardinality of the set of powered relays).

The optimisation problem 'Dynamically Powered Relays for a Flexible Optical Network' (DRP-FON) is, thus, defined as:

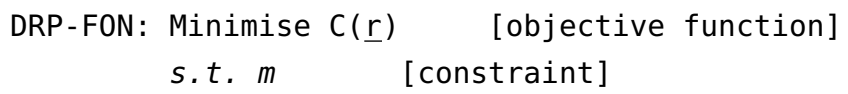

\section{Theorem. DRP-FON is NP-Complete.}

Proof. (informal) Intuitively, by direct reduction to the 'Minimum Dominating Set' (MDS) [19] (note, not MCDS [20], since the relays do not, themselves, have to form a connected core) and demonstration of polynomial certification (the full version follows).

\section{Related Algorithms}

Rapidly-varying transmission parameters, leading to changing values of $m$, will require frequent (re-)optimisation of DRP-FON, and re-minimisation of $C(\underline{r})$. Conventional linear and integer programming [21], 'branch-and-cut' [20], 'branch-and-price-and-cut' [22] methods, metaheuristics [14], or exhaustive search approaches [23] will be inappropriate. Not only is speed of the essence, but 
optimisation may also have to be performed on network devices with limited processing power. Instead, we base our approach on approximation algorithms for related problems in Section 2 [13] and, in particular, the 'ADD' algorithm [16] for optimisation of relay placement in wireless networks (without the connected core network requirement).

\section{A Fast Heuristic Solution}

Assuming the need for a FON configuration supporting a given value of $m$, a fast heuristic for DRP-FON is constructed as follows:

Define the hop matrix, $H$, as $H=\left(h_{i j}\right)$ where $\forall i j, h_{i j}=\mid\{a$ : link $a$ is in the shortest path between $i$ and $j\} \mid$ (the length of the shortest path, in hops, between nodes $i$ and $j)\left(h_{16}=4\right.$ in Figure 1, for example). The first subroutine, using Dijkstra's Shortest Path Algorithm [18] (or similar) [24], is then:

$\underline{A l g H}$ (Calculate the hop matrix):

$\overline{\forall i j}$, run DSPA to calculate $H=\left(h_{i j}\right)$

(Note that DSPA only runs once to calculate each row of the matrix, $H$, not individually for each element, $h_{i j}$.)

The second subroutine calculates the viability matrix, $V=\left(v_{i j}\right)$, defined by the existence of a path between each node pair satisfying $m$. For each $i j$ pair, $v_{i j}=T$ if the hop count between $i$ and $j$ is, at most, $m$, or a sequence of relays can be found between $i$ and $j$ with the hop count of each step between, at most, $m$ relays.

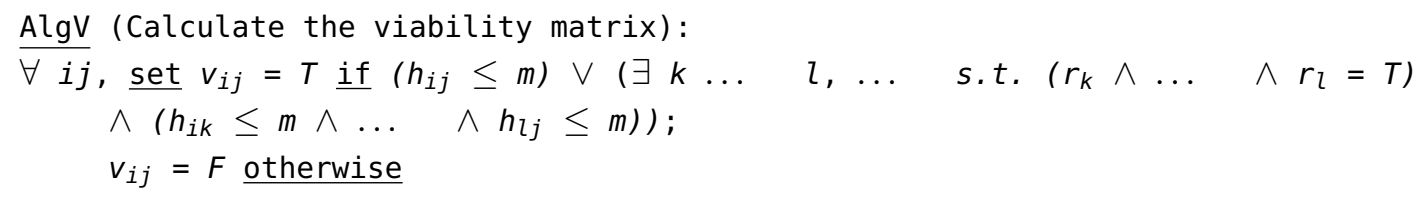

With this definition, we may now revisit the NP-completeness theorem:

Theorem. DRP-FON is NP-Complete.

Proof. DRP-FON is NP-hard since, for $m=1$, it reduces to the 'Minimum Dominating Set' (MDS) [15].

A certificate for DRP-FON can be verified in polynomial time by the following algorithm:

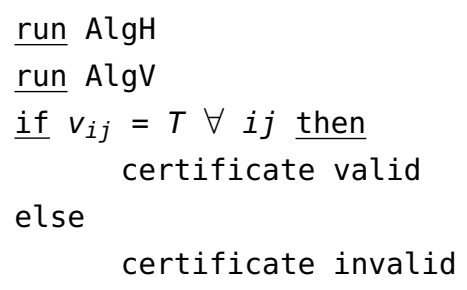

Returning to the solution, the complete algorithm starts by initialising all relays to be switched off (unpowered), then uses a greedy (limited local search) heuristic to switch relays on (power), one at a time. The relay to be newly powered is that which has a viable path (i.e., with or without intermediate relays), of a length no greater than $m$, to the largest number of other nodes, not currently acting as relays-thus, maximising the number of new nodes that are 'reached/spanned' by the extra relay. The algorithm terminates when the overall solution becomes viable, i.e., when paths between all node pairs (with or without intermediate relays) are viable. This includes the special case that the initial solution is viable without any relays. 


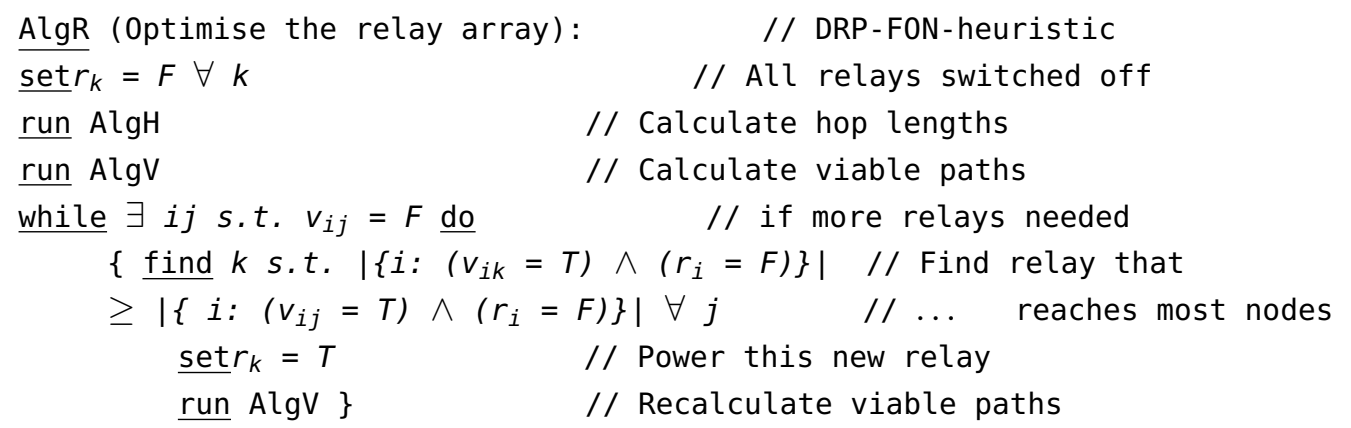

Note, once more, that the relays need not form a connected network. The DRP-FON heuristic, $\mathrm{AlgR}$, can be considered an extension of the greedy 'ADD' algorithm in [16] with the maximal metric for relay selection extended to include those nodes within a 'radius' of $m$ from each candidate location.

\section{Testing and Evaluation}

AlgR has polynomial complexity. In the initialisation part, switching off all relays has complexity $O(n)$. DSPA (AlgH) is bounded by $O\left(n^{3}\right)$ [18] and can generally be implemented as $O\left(n^{2}\right)$ [24]. Similarly, $\mathrm{AlgV}$ is $O\left(n^{3}\right)$, at worst, but $O\left(n^{2}\right)$ in practice. Anyway, this part is only executed once. The more significant non-deterministic while loop, in fact, iterates at most $n$ times (a maximum of $n$ nodes/relays to potentially switch on). Within it is a maximisation search of $O\left(n^{2}\right)$, giving an overall complexity of $O\left(n^{3}\right)$.

To evaluate AlgR, we use two standard test examples [25] and compare with a 'brute force' approach [23]. Figures 3 and 4 shows the standard 'US NSF' and 'COST-266 European' optical networks with distances ignored (since we initially only consider node adjacency). For COST-266, there are $2^{28}$ possible relay 'on/off' combinations, which is manageable for a simple exhaustive search algorithm with some patience. Table 1 compares $\mathrm{AlgR}$ with the known optimum generated by brute force. Each solution has been coded in C\# running on a standard desktop PC. AlgR ran within a tenth of a second in each case; the exhaustive search, considerably longer.

Table 1 shows the amount (number of powered relays) by which AlgR performs worse than the 'perfect' exhaustive search algorithm for different $m$ constraints (not all values of $m$ are meaningful: when $m$ reaches the length of the longest path between any nodes, for example, no relays are needed) It might be reasonable to expect the performance of the AlgR heuristic to improve with larger values of $m$ as smaller numbers of relays suffice, but this pattern does not emerge clearly in these two test instances.

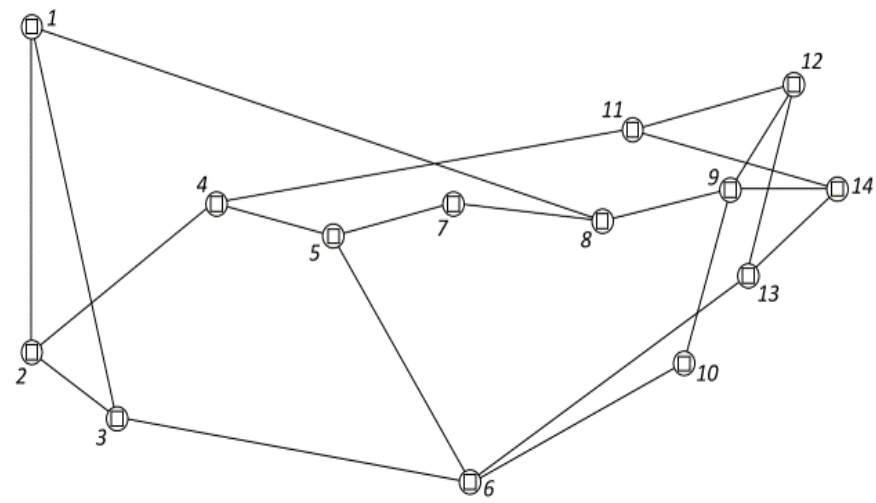

Figure 3. The US NSF network (topologically, not geographically, accurate). 


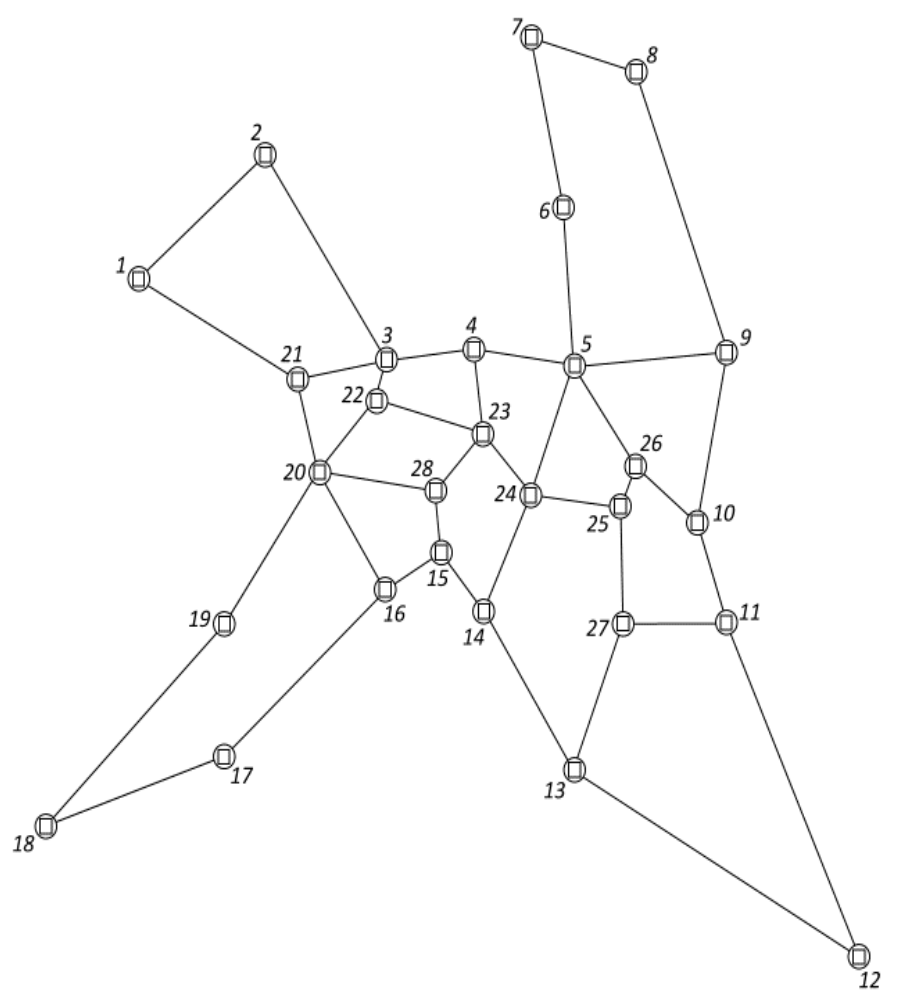

Figure 4. The COST-266 European network (topologically, not geographically, accurate).

Table 1. Comparing AlgR with known optima.

\begin{tabular}{ccc}
\hline$m$ & US NSF $^{\mathbf{a}}$ & COST-266 $^{\mathbf{a}}$ \\
\hline 1 & 1 & 2 \\
2 & 1 & 1 \\
3 & 1 & 2 \\
4 & 0 & 1 \\
5 & 1 & 0 \\
6 & - & 1 \\
7 & - & 0 \\
8 & - & -
\end{tabular}

${ }^{a}$ Extra relays in AlgR solution above optimum. - Trivial solution.

For these small examples, individual analysis may be specious; however, Table 1 represents a mean increase of $14 \%$ in $C(\underline{r})$ above the known optima over all sensible values of $m$ in both cases. This is comparable with (in fact, somewhat better than) similar examples in which a crude/fast (polynomial) heuristic replaces a complex/slow metaheuristic [26] or (exponential) exhaustive search [13]. However, additional testing is still clearly needed.

\section{Extensions and Generalisations}

DRP-FON has been defined, in this paper, for simplicity of the initial analysis, solution, and testing. There are a number of respects in which it may be too simple for widespread application. In particular, the following real-world extensions may be considered:

Ext1: Relays are only permitted at certain nodes: This is a likely extension to the basic model, reflecting restrictions on equipment installation at some locations or resulting from network management strategies, such as only installing regenerators at some (half, say) of all locations. It is 
easily dealt with by a simple restriction on the $\underline{r}$ array or, if necessary, a separate $\underline{p}$ 'permitted' array. The adaptation of $\mathrm{AlgV}$ and $\mathrm{AlgR}$ is then trivial.

Ext2: Use physical distances instead of hop counts: If regeneration limits are to be measured by cumulative distance, rather than merely hop count, then reachability becomes an issue of both fixed physical edge weight, $W=\left(w_{i j}\right)$ (the Euclidean distance between $i$ and $j$, for example), and the dynamically-changing limit, $m$, as transmission parameters vary. As a result, the viability matrix, $V$, is calculated by natural extension to $\mathrm{AlgH}$ and $\mathrm{AlgV}$, and $\mathrm{AlgR}$ remains essentially unaltered.

Ext3: Allow different transmission limits for each node pair: The basic model assumes the same changes in transmission parameters and, hence, $m$, across the FON. However, if transmission parameters are allowed to vary locally (independently) then the single value of $m$ will need to extend to a matrix of limits, $M$. Nevertheless, individual values, $v_{i j}$, of the viability matrix, $V$, can be calculated, without difficulty, using the appropriate element, $m_{i j}$ : $\mathrm{AlgV}$ and $\mathrm{AlgR}$ otherwise work as before.

Taking these three classes of extension together gives a new optimisation problem to be considered. Firstly, we define the permitted/present vector, $p$, as $p=\left(p_{k}\right)$ where $\forall k, p_{k}=T$ if a relay is allowed/installed at node $k ; p_{k}=F$ otherwise. $p$ is a physical network constraint for any node set. Then, as before, for any given solution, the relay vector, $\underline{r}$, is defined as: $\underline{r}=\left(r_{k}\right)$ where $\forall k, r_{k}=T$ if the relay at node $k$ is switched on (powered); $r_{k}=F$ otherwise.

Next, we include the physical distance (or any appropriate metric) by introducing the weight matrix, $W=\left(w_{i j}\right)$, where $w_{i j}$ represents the 'weight' of the link between $i$ and $j$ (if present). In the simplest case, $w_{i j}$ represents the Euclidean distance between the two nodes (Figure 5). However, other uses are possible to discourage the use of inappropriate paths, etc. [12].

Finally, we extend our transmission limit concept to allow local variation across the FON. On this basis, we define the maximum hop matrix, $M=\left(m_{i j}\right)$, where $m_{i j}$ is the maximum number of hops for which an optical signal can be transmitted, between nodes $i$ and $j$, without the need for regeneration by a relay.

As before, the cost of a solution, $\underline{r}$, is then the number of relays switched on (the cardinality of the set of powered relays) in any given configuration (now defined by both $M$ and $W$ ). $C(\underline{r})=\left\{\left\{k: r_{k}=T\right\} \mid\right.$.

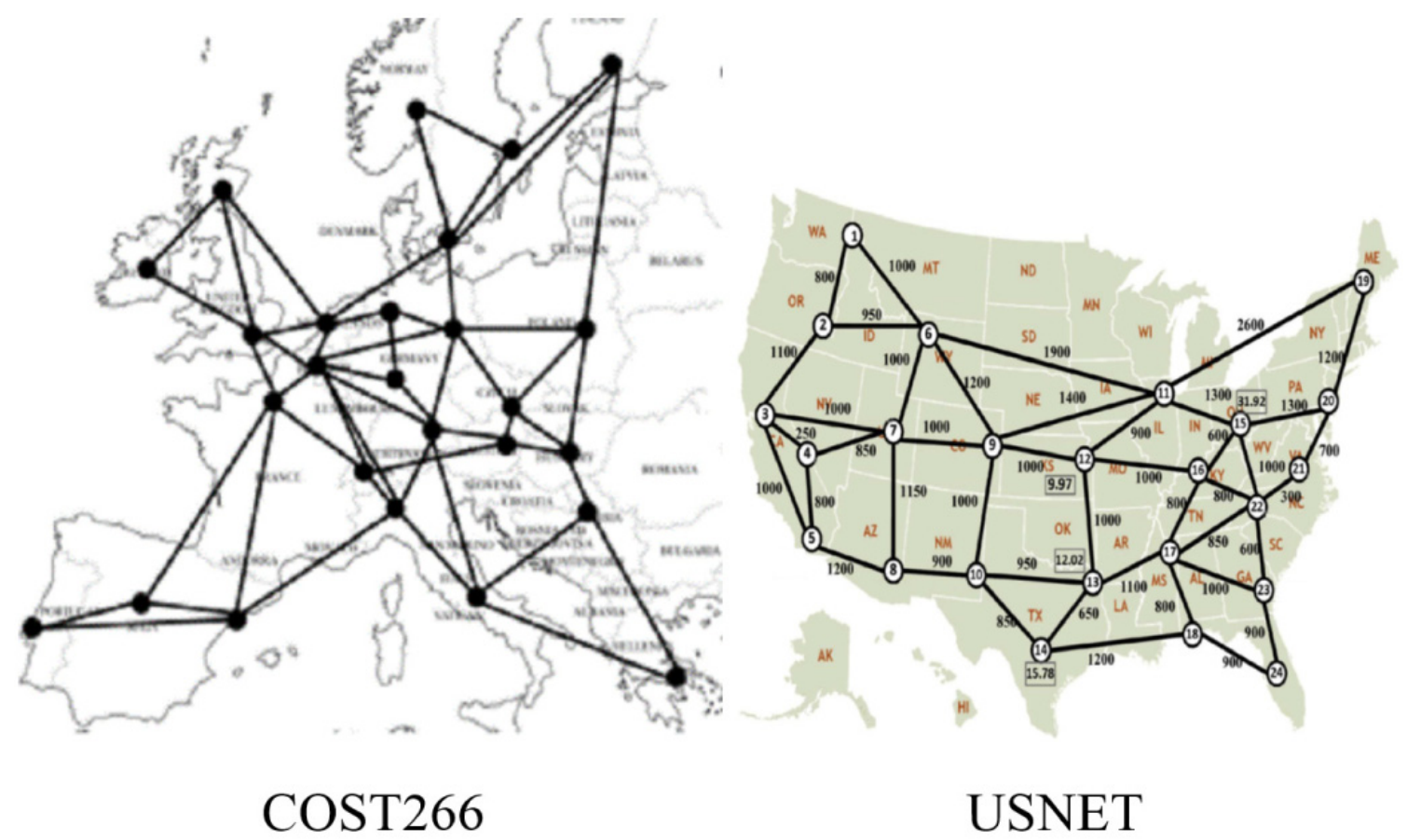

Figure 5. Euclidean (geographically accurate) COST-266 European and US NSF networks [7]. Note the additional links since the publication of the library: adapted from [25]. 
The new optimisation problem ‘Generalised Dynamically Powered Relays for a Flexible Optical Network' (GDRP-FON) is, thus, defined as:

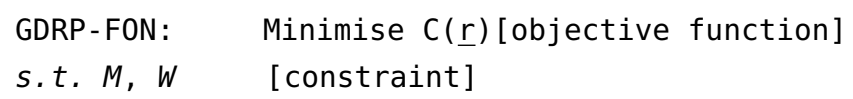

Theorem. GDRP-FON is NP-complete.

Proof. (informal) Intuitively, by direct reduction to DPR-FON and the demonstration of polynomial certification (the full version follows).

We now proceed to build a revised fast heuristic for GDRP-FON as follows:

Once again, define the hop matrix, $H$, as $H=\left(h_{i j}\right)$ where $\forall i j, h_{i j}=\mid\{a$ : link $a$ is in the shortest path between $i$ and $j\}$ I. However, now for the extended GDRP-FON problem, these hop counts have to take into account link weights, $W=\left(w_{i j}\right)$. Consequently, the first extended subroutine, $\mathrm{AlgGH}$, again using Dijkstra's Shortest Path Algorithm becomes:

AlgGH (Calculate hop matrix):

$\overline{\forall i j, \underline{\text { run }}} D S P A$ to calculate $H=\left(h_{i j}\right)$ using the weightings, $W=\left(w_{i j}\right)$ as SPA edge costs ( $W$ : input; $H$ : output) (note that DSPA still only runs once to calculate each row of the matrix, $H$, not individually for each element, $h_{i j}$. Additionally, although the $w_{i j}$ values define actual weightings, the shortest path (the result), in each case, need only be recorded in hops, $h_{i j}$ ).

The second subroutine, AlgGV, calculates the viability matrix, $V=\left(v_{i j}\right)$, which now has to be extended in two respects. In defining the existence of a path between each node pair $i j$, satisfying $m_{i j}$ (now a local value), it must also take into account whether a relay is present or permitted at each node $k$. For each $i j$ node pair, then, $v_{i j}=T$ if the hop count between $i$ and $j$ is at most $m_{i j}$ or a sequence of permitted relays can be found between $i$ and $j$ with the hop count of each step between relays $k$ and $l$ at most $m_{k l}$ (the limit is applied independently for each relay pair).

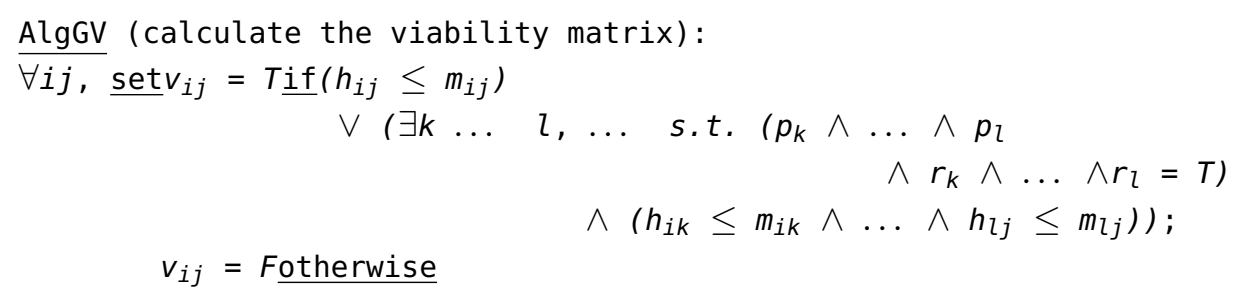

This now allows us to reconsider the NP-completeness proof:

Theorem. GDRP-FON is NP-complete.

Proof. GDRP-FON is NP-hard since, for $p_{k}=T \forall k, d_{i j}=h_{i j} \forall i j$ and $w_{i j}=m_{i j}=$ constant $\forall i j$, DRP-FON reduces to it.

As before, a certificate for GDRP-FON can be verified in polynomial time by the following algorithm:

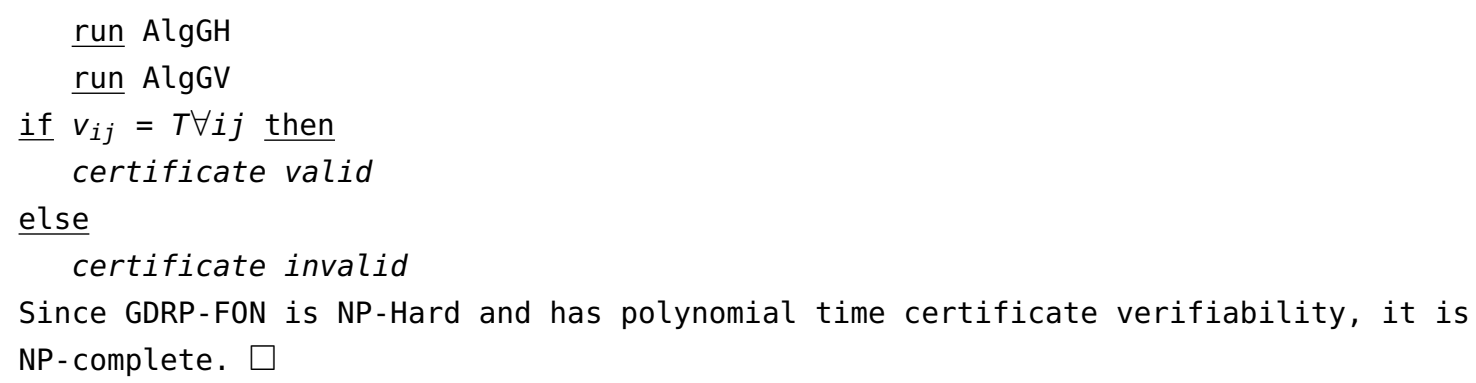


Again we return to the development of an equivalent fast heuristic for GDRP-FON, combining the newly-extended AlgGH and AlgGV subroutines. Once again, the complete algorithm starts by initialising all relays to be switched off, then uses a greedy heuristic to switch on permitted relays, one at a time. The relay to be newly-powered is that which (is permitted and) has viable paths to the largest number of other nodes, not currently acting as relays-thus maximising the number of new nodes that are 'reached/spanned' by the extra relay. Again, the algorithm terminates when the overall solution becomes viable, i.e., when paths between all node pairs (with or without intermediate relays) are viable.

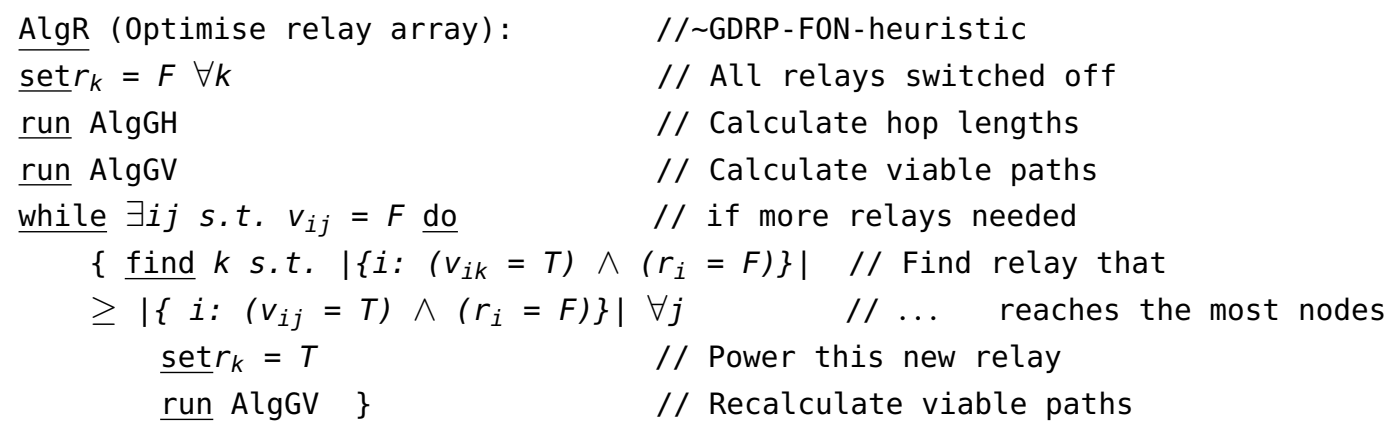

As before, the GDRP-FON heuristic, AlgGR, can be considered an extension of the greedy 'ADD' algorithm in [16]. Again, it deals with the special case of an initial configuration (and values of $M$ and $W$ ) requiring no relays. Additionally, as before, AlgGR is polynomial by a similar argument.

At this stage of the research, however, the AlgGR fast heuristic remains untested. Although AlgGR itself runs quickly, the brute force approach to finding the optimal comparison becomes intractable for GDRP-FON. A reliable branch-and-price-and-cut [22] or metaheuristic [21] will have to be developed.

\section{Conclusions and Future Work}

This concludes an introduction to the field. Taking into account recent advances in optical network technology, this work has introduced the newly-relevant DRP-FON relay management problem in its basic form, defined it formally, and proved its NP-completeness. A simple, fast heuristic, AlgR, has been presented and shown, by comparison with more computationally-expensive optimisation techniques, to give valid and reasonable results. Both the problem and fast solving heuristic have then been extended to a generalized case (GDRP-FON), also NP-complete, taking into account three real-world extensions. Finally, a generalized fast heuristic, AlgGR, has been proposed (but not tested).

Future work should now expand the testing of AlgR and AlgGR with larger examples. Additionally, other researchers are invited to develop improved heuristics for the simple and generalised DRP-FON and GDRP-FON problems, always taking into account, however, the practical constraints of real-world implementation, namely:

- The algorithm may have to run, and frequently re-run, within the limited operating environment of production network equipment, implying that:

- The algorithm should be of polynomial complexity in both space and time (probably no worse than $O\left(n^{3}\right)$ steps and $O\left(n^{2}\right)$ memory).

A final consideration is that, in some practical implementations, the algorithm may have to run in distributed, rather than centralised, form: i.e., independently—but cooperatively [27]—on each network device. This is non-trivial.

Conflicts of Interest: The author declares no conflict of interest. The founding sponsors had no role in the design of the study; in the collection, analyses, or interpretation of data; in the writing of the manuscript, and in the decision to publish the results. 


\section{References}

1. Yetginer, E.; Karasan, E. Regenerator Placement and Traffic Engineering with Restoration in GMPLS Networks. Photonic Netw. Commun. 2003, 6, 139-149. [CrossRef]

2. Jinno, M.; Takara, H.; Kozicki, B.; Tsukishima, Y.; Sone, Y.; Matsuoka, S. Spectrum-Efficient and Scalable Elastic Optical Path Network: Architecture, benefits, and enabling technologies. IEEE Commun. Mag. 2009, 47, 66-73. [CrossRef]

3. Yildiz, B.; Karasan, O.E. Regenerator Location Problem in Flexible Optical Networks. Available online: http:/ / www.optimization-online.org/DB_HTML/2014/11/4659.html (accessed on 31 December 2015).

4. Yildiz, B.; Karasan, O.E. Regenerators as Hubs. Trans. Res. Part B 2014, 94, 1823-1830.

5. Essiambre, R.-J.; Kramer, G.; Winzer, P.J.; Foschini, G.J.; Goebel, B. Capacity Limits of Optical Fiber Networks. J. Light. Techbol. 2010, 28, 662-701. [CrossRef]

6. Sen, A.; Murthy, S.; Bandyopadhyay, S. On Sparse Placement of Regenerator Nodes in Translucent Optical Networks. In Proceedings of the 2008 IEEE Global Telecommunications Conference, New Orleans, LA, USA, 30 November-4 December 2008; pp. 1-6.

7. Le, H.-C.; Hasegawa, H.; Sato, K.-I. Performance Evaluation of Large-scale Multi-stage Hetero-granular Optical Cross-connects. Opt. Express 2014, 22, 3157-3168. [CrossRef] [PubMed]

8. Jinno, M.; Kozicki, B.; Takara, H.; Watanabe, A.; Sone, Y.; Tanaka, T.; Hirano, A. Distance-Adaptive Spectrum Resource Allocation in Spectrum-Sliced Elastic Optical Path Network: Topics in optical communications. IEEE Commun. Mag. 2010, 48, 138-145. [CrossRef]

9. Tomkos, I.; Palkopoulou, E.; Angelou, M. A Survey of Recent Developments in Flexible/Elastic Optical Networking. In Proceedings of the 2012 14th International Conference on Transparent Optical Networks, Coventry, UK, 2-5 July 2012; pp. 1-6.

10. Chen, S.; Ljubic, I.; Raghavan, S. The Regenerator Location Problem. Networks 2009, 55, 205-220. [CrossRef]

11. Yang, X.; Ramamurthy, B. Sparse Regeneration in Translucent Wavelength-Routed Optical Networks: Architecture, network design and wavelength routing. Photonic Netw. Commun. 2005, 10, 39-53. [CrossRef]

12. Houlden, N.; Grout, V.; McGinn, J.; Davies, J. Extended End-to-End Cost Metrics for Improved Dynamic Route Calculation. In Proceedings of the 6th International Network Conference, Plymouth, UK, 11-14 July 2006.

13. Grout, V.; Davies, J.N.; McGinn, J. An Argument for Simple Embedded ACL Optimisation. Comput. Commun. 2007, 30, 280-287. [CrossRef]

14. Morgan, M. Optimisation Techniques for Wireless Networks. Ph.D. Thesis, Glyndŵr University, Wrexham, UK., 2010.

15. Morgan, M.; Grout, V. Spanning Tree Objective Functions and Algorithms for Wireless Networks. In Proceedings of the 2006 IEEE Sarnoff Symposium, Princeton, NJ, USA, 27-28 March 2006; pp. 1-4.

16. Grout, V. Principles of Cost Minimisation in Wireless Networks. J. Heuristics 2005, 11, 115-133. [CrossRef]

17. Lucena, A.; Maculan, N.; Simonetti, L. Reformulations and Solution Algorithms for the Maximum Leaf Spanning Tree Problem. Comput. Manag. Sci. 2010, 7, 289-311. [CrossRef]

18. Dijkstra, E.W. A Note on Two Problems in Connexion with Graphs. Numer. Math. 1959, 1, 269-272. [CrossRef]

19. Garey, M.R.; Johnson, D.S. Computers and Intractability: A Guide to the Theory of NP-Completeness; Freeman: San Francisco, CA, USA, 1979.

20. Gendron, B.; Lucena, A.; da Cunha, A.S.; Simonetti, L. Benders Decomposition, Branch-and-Cut and Hybrid Algorithms for the Minimum Connected Dominating Set Problem; Technical Report; Interuniversity Research Centre on Enterprise Networks, Logistics \& Transportation: Montreal, QC, Canada, 2012.

21. Morgan, M.; Grout, V. Metaheuristics for Wireless Network Optimisation. In Proceedings of the 2007 Third Advanced International Conference on Telecommunications, Morne, Mauritius, 13-19 May 2007; pp. 1-4.

22. Barnhart, C.; Hane, C.A.; Vance, P.H. Using Branch-and-Price-and-Cut to Solve Origin-Destination Integer Multicommodity Flow Problems. Oper. Res. 2000, 48, 318-326. [CrossRef]

23. Grout, V. Initial Results from a Study of Probability Curves for Shortest Arcs in Optimal ATSP Tours with Application to Heuristic Performance. In Proceedings of the 20th British Combinatorial Conference, Durham, UK, 10-15 July 2005; p. 93.

24. Wagner, D.; Willhalm, T. Speed-up Techniques for Shortest-Path Computations. In Annual Symposium on Theoretical Aspects of Computer Science; Springer: Berlin/Heidelberg, Germany, 2007; pp. 23-36. 
25. Hulsermann, R.; Betker, A.; Jager, M.; Bodamer, S.; Barry, M.; Spath, J.; Gauger, C.; Kohn, M. A Set of Typical Transport Network Scenarios for Network Modelling. ITG Fachbericht 2004, 182, 65-72.

26. Morgan, M. An Ant Colony Approach to Regenerator Placement with Fault Tolerance in Optical Networks. In Proceedings of the 2015 7th International Workshop on Reliable Networks Design and Modeling, Munich, Germany, 5-7 October 2015; pp. 85-91.

27. Grout, V.; Houlden, N.; Davies, J.; McGinn, J.; Cunningham, S. A Unified Framework for Optimal Routing. In Proceedings of CSSI International Conference System Integration in Integrated Europe (ISIE), Liberec, Czech Republic, 8-9 November 2004.

(C) 2017 by the author. Licensee MDPI, Basel, Switzerland. This article is an open access article distributed under the terms and conditions of the Creative Commons Attribution (CC BY) license (http://creativecommons.org/licenses/by/4.0/). 